In press: Decision (ISSN: 2325-9965)

\title{
Conjectures on Science and Rationality: Commentary on Fiedler, Salmen, \& Prager (2022)
}

\author{
David R. Mandel \\ Defence Research and Development Canada
}

\begin{abstract}
With their spotlight on Laplace's inductive rule of succession, Fiedler et al. (2022) revisit important questions about what constitutes rationality and how rationality can be well tested in experimental research. In commentary on their article, I propose that Popper's evolutionary epistemology can help address both questions.
\end{abstract}

Fiedler et al. (2022) invite us to think about two big questions. What is rationality? And, what serves as good experimental evidence for rationality or its converse? These questions are not unrelated as both may be suitably answered by Popper's (1962) evolutionary epistemology, which regards science as a process of problem definition, conjecture, refutation and evolution to new conjectures and, eventually, to new problems. To its credit, Popper's epistemology is anti-utopian and anti-authoritarian. There are no final solutions to deep problems. No inescapable proofs of truth, but rather, a trail of refutations with the expectation (if not the outright hope) of still more to follow. The best scientists ensconced in this tradition will want even their best theories to join the trail, if (but only if!) for good reason, since only in this way can better collective explanations of the physical world be wrought. This approach demands exactingness in experimental tests of alternative explanations, as Feynman so eloquently described in his 1974 Caltech commencement address on what he called cargo-cult science. ${ }^{1}$ Fiedler et al. are therefore quite right that anemic empirical tests simply won't do. Their spotlight on people's adherence to Laplace's rule of succession is illuminating precisely because it clearly exposes this requirement.

Lapses of exactingness do not imply that the conclusions drawn from experimental tests are incorrect, but because such lapses trigger failures to discriminate between competing explanations, they increase the probability of accepting false conclusions about what has happened. Therefore, they do not advance science but rather dilute it with inconclusive results. Nor is there anything in exactingness that increases the chances of a particular conclusion with respect to rationality claims. In Fiedler et al., increasing exactingness reveals that earlier conclusions about rationality are unwarranted. By comparison, my earlier work on framing (Mandel, 2014) demonstrated that increasing exactingness by eliciting people's linguistic interpretations of numeric quantifiers along with their choices calls into question the assumption that framing effects based on the highly-studied "Asian disease problem" (ADP, Tversky \& Kahneman, 1981) provide evidence of irrationality. If a majority of individuals (as I found), interpret the numeric quantifiers in the statements " 200 will be saved" and "400 will die" as lower bounds, this suffices to invalidate the claim that the alternative descriptions given in ADP experiments are extensionally equivalent. Clearly, it is not evidence of labile risk attitudes nor incoherence due to violation of the description invariance principle to prefer saving at least 200 out of 600 versus a $1 / 3$ chance of saving 600 , while preferring the latter gamble to a prospect that offers at least 400 deaths in 600. Due to inexactness, the ADP unfortunately proves nothing about human rationality, in general. In fact, without the guarantee of extensional equivalence, the ADP and the scores of problems modeled on it do not test for framing effects at all. Exactingness always matters.

1 A copy is available from https://wwwphy.princeton.edu/ steinh/CargoCult.pdf. 
If Popper's evolutionary epistemology is a good model for science, perhaps it is also a good model for individual and collective rationality. After all, is it not the epitome of rationality to seek an increase in our understanding of ourselves, each other, aspects of our environment and, ultimately, the entirety of reality? Is there a more rational way to do so than through problem definition, conjecture, honest attempts at refutation (i.e., regardless of by whom the ideas originated), and revision of explanation as the implications of new evidence call for? And though such a rational agent may aspire to truth-seeking, note that this conception of rationality eschews equating it with correspondence with reality. By brute application of the correspondence criterion, we would be forced to conclude that Einstein was more rational than Newton for his explanation of physical reality is undoubtedly better, but such a claim is utterly foolish. In the terms Fiedler et al. use, it serves as an existence proof of the failure of correspondence as a basis for rationality. Brute application of coherence does no better as a basis for rationality. A Bayesian is encouraged but not obliged to follow Cromwell's rule (Lindley, 1982). The Bayesian for whom no amount of evidence can overturn a certain prior (namely, of either $p=0$ or $p=1$ ) is fully consistent, yet we would not regard him as the epitome of rationality.

I agree with Fiedler et al. that experiments should be exacting, but we must also ask to what end is such work applied. If Fiedler et al. had instead found a pattern of results predicted by the sound application of the rule of succession, what then? Would adherence to this rule have demonstrated that people are rational? Only in a narrow sense that the authors themselves appear to reject (e.g., as they stated, "Scientists should thus refrain from useless attempts to operationalize the idealized competence of rationality as incidental performance on arbitrarily selected tasks..."). But if we accept that, is it not true that their actual results do not speak to violations of rationality either. To be sure, the findings speak to the correct application of the rule of succession by individuals, most of whom have probably never been taught the rule. Why then should their adherence to it be regarded as a test of rationality any more than testing whether they can correctly determine the circumference of a circle from its diameter? At least $2 \pi \mathrm{r}$ is universal. Laplace's rule is generally inapplicable since, as Fiedler et al. noted, the rule of succession does not apply if the sequence of outcomes is not stochastically independent.

Finally, what of Bertrand Russell's poor chicken who diligently follows at least the spirit of the

rule of succession? This, of course, refers to Russell's (1912) famous quote about a chicken, who in the true spirit of inductivism, comes to believe in the benevolence of the farmer who feeds him daily until the day the farmer instead rings his neck. The sad fate of farm chickens are all the same regardless of their epistemologies, but let's imagine that Russell's chicken is endowed with a fighting chance of escape and eventual freedom, and then put yourself in the chicken's place. Would you choose to be a Laplacian-Bayesian chicken or a Popperian chicken? Induction would squander your chances for survival, and that hardly seems rational even in a narrow sense. Most species (i.e., upwards of 99\%) that have ever lived on Earth are now extinct. Our own species is the last survivor of the genus Homo. If collective rationality means anything, then surely it must help us escape extinction-level events.

\section{References}

Fiedler, K., Salmen, K., \& Prager, J. (2022). Toward stronger tests of rationality claims: Spotlight on the rule of succession. Decision.

Lindley, D. V. (1982). The Bayesian approach to statistics. In J. Tiago de Olivera (Ed.), Some recent advances in statistics (pp. 65-87). Academic Press.

Mandel, D. R. (2014). Do framing effects reveal irrational choice? Journal of Experimental Psychology: General, 143, 1185-1198. doi:10.1037/a0034207

Popper, K. R. (1962). Conjectures and refutations: The growth of scientific knowledge. Basic Books. 
Russell, B. (1912). The problems of philosophy. Oxford University Press.

Tversky, A., \& Kahneman, D. (1981, January 30). The framing of decisions and the psychology of choice. Science, 211, 453-458. doi:10.1126/science.7455683 